\title{
Development of A Microhand using Direct Laser Writing for Indirect Optical Manipulation
}

\author{
Ebubekir Avci ${ }^{1}$ and Guang-Zhong Yang ${ }^{2}$
}

\begin{abstract}
In this paper, we propose manipulation ability extension of the optical tweezers by developing microhands, which are to use as end-effectors of the laser beam. First, three different 3D micro-scale handles are designed, then manufactured by the two-photon polymerization method with nano-scale resolution of $100 \mathrm{~nm}$. Second, printed microhands are manipulated by multi-spot laser beam which traps and manipulates numerous objects simultaneously. Third, where direct trapping of the target object is not possible due to target objects' features such as size, shape, material, index of refraction, etc., indirect manipulation of the target microobjects is achieved by using the microhands as an extension of optical tweezers. Finally, three different microhand designs are compared in terms of speed and success rate. Furthermore, suitability of different shapes of microhandles against common usage of spherical shape is discussed.
\end{abstract}

\section{INTRODUCTION}

To control and manipulate tiny objects ranging in size from tens of nanometres to tens of micrometres, optical tweezers are suitable instruments, notably in confined spaces [1], [2]. Optical tweezers can move particles using the forces -ranging from femtonewtons to nanonewtons- produced by a strongly focused laser beam [1]. In 1970, the moving of a dielectric particle was achieved for the first time using two laser beams [3]. Later, in 1986, the possibility of microobject trapping using one laser beam was verified [4].

Recently, optical trapping has gathered much interest from researchers working in the bio-medical field and it has been used for a number of different purposes due to its precise and non-invasive manipulation ability [5]. Intracellular surgery -modifying the chromosomes of living cells- has been achieved [6]. Single-molecule DNA stretching and twisting have been realized to study biological processes such as DNA replication and transcription [7]. Cell sorting, classification and cell fusion tasks have been undertaken successfully [8], [9]. The automatic transportation of multi-cells has been achieved [5]. Direct measurement of cell protrusion force to characterize the mechanism of cell migration utilizing a robot-aided optical tweezer system has been accomplished [10]. The dynamic and static micromechanical properties of human embryonic stem cells-derived cardiomyocytes have been measured via optical tweezers to study mechanical and contractile defects in heart diseases [11].

Optical tweezers have been used for non-contact manipulation of dielectric particles successfully. However, this method

\footnotetext{
${ }^{1}$ Ebubekir Avci is with Mechatronics Department, School of Engineering and Advanced Technology, Massey University, NZ. e.avci@massey.ac.nz

${ }^{2}$ Guang-Zhong Yang is with The Hamlyn Centre for Robotic Surgery, Imperial College London, UK
}

has the following drawbacks. First, the force that emerges from the focused laser beam is not enough to manipulate large size microobjects. Second, material of the target object should be dielectric and should possess appropriate refraction index to be trapped. Third, spherical shapes are suitable to be manipulated but if the target object has an asymmetric shape, then the trapping of the target becomes an arduous task [12]. Fourth, some biological objects might be damaged by laser beam heat [13]. Thus, the trapping ability of the optical tweezers is limited with target objects' size, shape, material, index of refraction, etc.

To improve manipulation abilities of optical tweezers, most studies have focused on sculpting the light field, only a few studies have checked shaping the object itself [14], [15]. Maruyama et al. successfully measured $\mathrm{pH}$ value of the yeast cell by manipulation of a circular gel-microbead, which surrounds the target cell [16]. Phillips et al. raster scanned the surfaces of biological particles by an optically trapped cigar-shaped probe as a novel imaging technique [17]. Tan et al. manipulated two beads attached to a cell by optical traps during cell stretching to obtain the mechanical response of the cardiomyocyte cell [11]. Fukuda et al. manipulated a microtool with temperature sensing ability to measure the temperature change of virus infected cells, by holographic optical tweezers [18]. In all these studies, instead of applying optical traps to the target object, a microtool has been trapped by laser beam to analyze the target object. Furthermore, in 2007, Arai et al. attached gel microspheres to lipid nanotubes to be manipulated by optical tweezers as the feasible maximum force emerging from optical tweezers was not sufficient for direct trapping of rod-shape nanotubes [12]. In 2010, Maruyama et al. produced triangle-shaped microtools consisting of three microbeads as an end-effector of optical tweezers so as not to damage the biological cells [13]. However, in the study, there was no demonstration of indirect object manipulation. In 2014, Thakur et al. proposed automated indirect pushing of biological cell in 2D which was very effective for less exposure of light but takes long time to complete the task due to lack of robust control of target cell [19]. All the above mentioned studies have contributed to the manipulation ability extension of optical tweezers using the concept of indirect manipulation.

In this paper, we propose further enhancement of the optical tweezers' manipulation ability by focusing on the design of various microhands, which are used as an endeffector of the laser beam. First, a 3D micro-scale handle is designed and manufactured by the two-photon polymerization (2PP) method [20]. By applying a focused laser 
beam on to a transparent liquid/gel resin which is sensitive to light, it becomes feasible to cure and polymerize with nano-scale resolution $(100 \mathrm{~nm})$. The focus of the laser is moved to scan the resin at specific points according to a preprogrammed path and any 3D shape in nano/micro scales can be printed creating a microstructures. The microhand produced is transferred on to a glass substrate to be used under optical tweezers by using nanomanipulators under scanning electron microscopy (SEM). Second, printed microhands are manipulated by multiple-spot optical tweezers that can trap and manipulate numerous objects simultaneously by scanning predetermined points rapidly $(60 \mathrm{kHz})$. Third, where direct trapping of the target object is not possible, manipulation of the target microobjects is achieved by using the microhands as an extension of optical tweezers. Using this method, therefore, manipulability of the target object by the highly focused laser beam depends less on the feature of the target microobjects. Finally, direct and indirect manipulation methods are compared and suitability of each design is discussed.

\section{MATERIALS AND METHODS}

\section{A. DESIGN AND FABRICATION OF MICROHANDS}

1) DESIGNING: The microhand should possess following features to be used as an end effector of optical tweezers. A minimum size of $1 \mu \mathrm{m}$ for the microhand is appropriate as it should be seen clearly via optical microscope (maximum resolution is about $0.2 \mu \mathrm{m}$ ). Upper limit for the size can be decided considering the maximum force emerging from laser beam ( enough to manipulate tens of $\mu \mathrm{m}$ ) and visible space under 100X magnification ( $40 \mu \mathrm{m} \times 50 \mu \mathrm{m})$. To control the microhands under visible space, $20 \mu \mathrm{m}$ is decided as an upper size limit. Therefore, the range for the size of the robot is $1-20 \mu \mathrm{m}$. As a material, photoresist (Nanoscribe, IP-G) is suitable for the refractive index of $n: \sim 1.5$ where $n$ for water is 1.33. As the refractive index of the object should be bigger than surrounding medium, the microhand material is suitable for optical trapping. As material is dielectric and bio-compatible, can be used for various bio-application. The holding points of microhands should be in spherical shape to be manipulated at ease.

2) PRINTING: The microhand is produced by solidifying the liquid polymer through directly scanning a laser along predetermined path as shown in Fig. 1. This method achieves high resolutions $(100 \mathrm{~nm})$ by taking advantage of two photon photo-polymerization (2PP).

3D printing system (Nanoscribe $\mathrm{GmbH}$ ) has following features. Laser beam was produced by femtosecond fiber laser source, which has wavelength of $780 \mathrm{~nm}$, pulse length of $100 \mathrm{fs}$ and repetition rate of $80 \mathrm{MHz}$. The produced laser beam was conveyed to the sample through mirrors, lenses and finally 100x oil-immersion objective. Sample, which consists of glass substrate and photoresist, controlled by motorized stage for coarse motion and piezo stage for the fine motion. An optical microscope (Zeiss) was used to visualize the process. The 3D microhand structure was generated in a layer-by-layer format. Galvanometric mirror scanner was

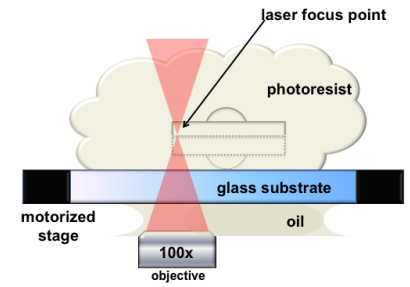

Fig. 1. Direct Laser Writing of the designed structure

used for $\mathrm{x}-\mathrm{y}$ directions (horizontal surface). Movement on the $\mathrm{z}$-axis was achieved using a piezo stage to change focus point of laser beam in vertical direction.

The detail of the printing process is as follows.

1) Cleaning of a coverslip to use as a glass substrate (Acetone, Isopropanol, DI water respectively).

2) A simple drop casting of the photoresist (IP-L 780) on to coverslip (spin coat and prebaking are not required for IP-L 780).

3) Approaching to sample and automatic finding of the interface between glass and photoresist by utilizing the sudden change of refractive index.

4) Printing the microhand using $2 \mathrm{PP}$ method

5) Development of the printed structure using ether acetate (PGMEA) for $40 \mathrm{~min}$, then a short bath using Isopropanol.

6) Using nitrogen gas stream for gentle dry of the microhand that adheres to the coverslip.

After drying the sample, the microhand was checked under SEM. For printing the microhand, power of $50 \mathrm{~mW}$ found an optimum parameter considering $0.1 \mu \mathrm{m}$ slicing (each layer) and $0.2 \mu \mathrm{m}$ hatching distance. In the case of lower power $(<40 \mathrm{~mW})$, curing of photoresist was not possible and for higher powers ( $>60 \mathrm{~mW})$, instead of curing, bubbling occurred. $10^{4} \mu \mathrm{m} / \mathrm{s}$ was the feasible maximum scanning speed; thus, printing of each microhand took under 5 minutes.

3) TRANSPORTATION: While printing the microhand on coverslip, the structure should adhere to the glass substrate to stay stable during the development (to be not washed away with other liquid of the remaining photoresist). Then, microhandle should be detached from the coverslip and transported to the optical tweezers workspace for actual usage as a robotic end effector. Here, transportation of these tiny objects is a critical issue [18].

The microhands were detached from the coverslip and moved on to a microscope slide which is to be placed under optical tweezers. Nanomanipulators (Kleindiek, NanoWorkstation) were used for detachment and transportation tasks. To follow entire procedure, sample was places inside the SEM (Tescan, FERA3 XM). The coverslip which possess the microhands and a microscope slide which is the destination of microhands are placed side to side under SEM.

To conduct an appropriate manipulation method, the effective forces in microscale should be considered. As length(d) of objects decrease from visible-scale to small-scale, surface 


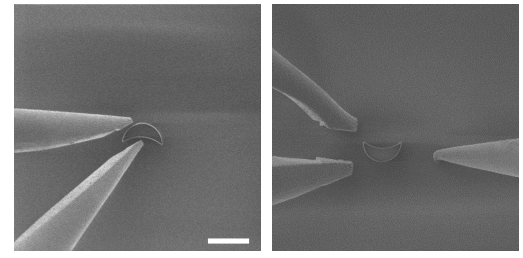

Fig. 2. Grasping the microhand (left), releasing the microhand using microprobe, scale: $10 \mathrm{um}$ (white bar: $10 \mu \mathrm{m}$ ).

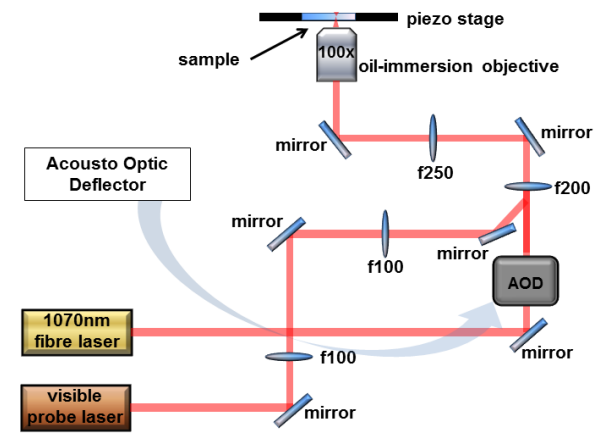

Fig. 3. Optical tweezers configuration.

forces $\left(d^{2}\right)$ begin to dominate volumetric forces $\left(d^{3}\right)$. Gravitational forces are proportional to the structure volume whereas adhesion forces (van der Waals forces, electrostatic forces, and surface tension forces) are proportional to the structure surface, adhesion becomes more effective than gravitational forces in the microscale [21]. Therefore, handling of microobjects is easier than releasing them. One microprobe is sufficient to handle a $30 \mu \mathrm{m}$ microtool due to adhesion forces. However, the effectiveness of adhesion forces is inversely proportional to the size of microobjects. Hence, releasing of smaller size microobjects is more difficult in the absence of a suitable method. Grasping of the microhand for detachment and transportation was carried out using microgriper (Kleindiek, MGS2-EM) as seen in Fig. 2 (left). After transportation, releasing of the microhands was realized using the microgripper with a microprobe (Kleindiek, LCMK-EM) as it has a small contact area with the object; this makes adhesion forces less effective as seen in Fig. 2 (right).

Before putting microscope slide, which has microhands on it, under optical tweezers, two drops of aqueous solution consisting DI water and $0.5 \%$ surfactant (Tween 20) are added on the microhands. DI water provides required medium for optical trapping where the surfactant prevents the microhands from sticking each other and glass surface. After preparing the sample, it was placed under optical microscope for optical manipulation.

\section{B. OPTICAL MANIPULATION SYSTEM}

For the micro-scale objects, trapping and manipulation by laser beam is plausible and can be explained with momentum conservation principle [22]. Dielectric micro particles under laser beam behaves in the same way with lens, refracting the

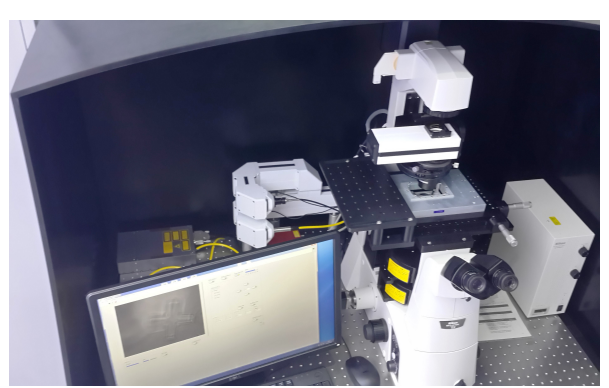

Fig. 4. Optical tweezers system.

light. Thus, direction of the photon momentum is changed and equal but opposite momentum change for the particle occurs. If the microobject is at the center of the focus, then the refraction of the light rays on the particle is symmetrical, and this lateral force on the object is zero. Therefore object is stable at the center. If the object move away from the center of the light, then the net force on the object, pull the particle back to the center of the light beam due to larger momentum change of more intense beams (at center of the light) compared to less intense beams (out of center). Once the particle is trapped by the laser beam, then manipulation of particle is possible in two ways, moving the light spot and moving the substrate by motorized stage. Manipulation of the object in aqueous medium acts as a damping force and helping for a stable manipulation. The particle shape is one of the significant parameters for the emerging force from laser beam in terms of direction and amount. Therefore, spherical and symmetrical shapes are easier to control under optical tweezers [14]. A few milliwatts is sufficient to trap and control microparticles.

To trap and manipulate microobjects, an optical tweezers (Elliot Scientific, E3500) integrated to an optical microscope (Nikon, ECLIPSE Ti) was used as seen in Fig. 3 and Fig. 4. There were two kinds of laser sources in the system: a high power fibre laser to manipulate particles and low power fibre laser for probing the beam. For trapping and manipulation purposes, Ytterbium fibre laser (YLM-10-LPSC), which emits $1070 \mathrm{~nm}$ wavelength light with a power of up to 10.6 watts (this is the optical output, the power reaches to the particle is around tens of $\mathrm{mW}$ ) was utilized. As shown in Fig. 3, laser beam was conveyed to the sample through mirrors, lenses and 100x oil immersion objective (Nikon, Plan Apo Oil).

To achieve multi-spot traps of laser beam, 2-axis AcoustoOptic Deflector (AOD) (IntraAction Corp., DTD-274HA6) was implemented. Therefore, manipulation of tens of microobjects simultaneously is feasible [23]. AOD manipulates position of the laser beam by altering the acoustic frequency. Frequency of the AOD was $60 \mathrm{kHz}$.

With the above-mentioned optical system, after trapping the target objects, manipulation can be done in passive and active ways. Passive method is the using fine motion stage (piezo actuated) to move the substrate in $\mathrm{X}-\mathrm{Y}-\mathrm{Z}$ axis. Hence, relocation of the object can be done by changing the position 

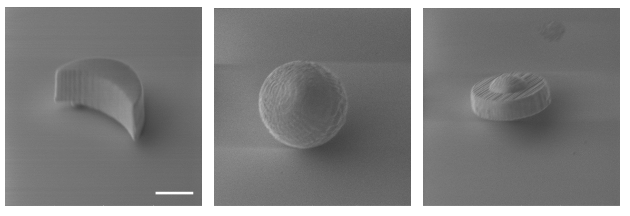

Fig. 5. Three different microhand designs, from left to right : the halfcylinder, the sphere and the cylinder with sphere (white bar: $5 \mu \mathrm{m}$ ).
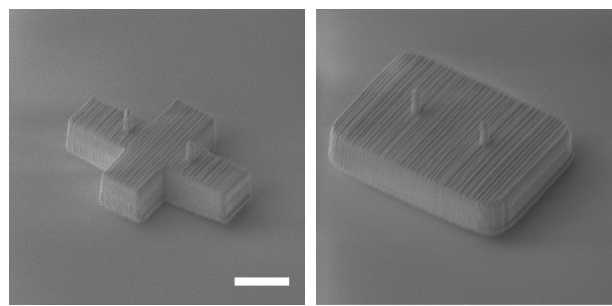

Fig. 6. Large size, non-spherical targets, (white bar: $5 \mu \mathrm{m}$ ).

of the substrate. In the active method, after trapping the object, laser beam can be steered using AOD. Thus, the target object can be moved from one point to another in the visible space of the microscope.

1) MANIPULATION APPROACH: As explained above, the plausible range for the microhand size is 1 to $20 \mu \mathrm{m}$. $10 \mu \mathrm{m}$ was chosen as the longest side of the each design. Three different designs were developed as seen in Fig. 5. The first design was half-cylinder shape, as it appeared like a microhand and grasping of microobjects would be easier with this end effector. The second microhand was sphere shape as trapping spherical shape is easier. The third design was the cylinder with sphere shape as a combination of the spherical and cylindrical shapes.

Aim of the study is to manipulate different microobjects which are not possible to be manipulated directly by optical tweezers. Therefore, two different large microobjects $(15 \mathrm{x}$ $20 \mu \mathrm{m}$ size was chosen as manipulation of bigger than this size would be difficult to be seen under 40 x $50 \mu \mathrm{m}$ size visible workspace) are designed to represent the challenging target objects. These objects are not spherical which makes direct manipulation an arduous task. First target object was a cross shape and the second one was rectangle as seen in Fig. 6.

Manipulation approach to target objects using microhands as end effectors of the optical tweezers is as follows. First, target objects and microhands were placed under the visible space of the microscope. Second, two microhands were trapped by multi-spot optical tweezers firmly. Third, two microhands approached to the target object from two opposite sides to grasp it in a balanced manner. Finally, transportation to a specific location was realized. Same procedure was carried out for the rotation of target objects. In this case, instead of relocating the target, spinning the target object was conducted which is an example of changing orientation of the target object in 2D.

\section{RESULTS AND DISCUSSION}

To check suitability of the designed microhands to be used as end effectors of optical tweezers, manipulation and rotation tasks were carried out for each microhand. After this, manipulation of large size microobjects using microhands was realised. Then, performance of each microhand was compared.

\section{A. THE MANIPULABILITY OF THE MICROHANDS}

The dynamic equation of the lateral motion of a microsphere is as follows [24].

$$
m \ddot{x}=F_{\text {trap }}(x)+F_{\text {drag }}
$$

$m$ is mass of the microsphere and $x$ is the position (the distance between target object and centre of laser beam). $F_{\text {trap }}(x)$ is the optical trapping force and $F_{\text {drag }}$ is the viscous drag force. Optical trapping force can be defined as:

$$
F_{\text {trap }}(x)=k x
$$

where $k$ is trapping stiffness constant. Viscous drag force can be defined using stokes' law as:

$$
F_{\text {drag }}=-6 \pi \eta r \dot{x}
$$

Where $\eta$ is viscosity, $r$ is radius of microsphere and $\dot{x}$ is the speed of the microsphere. For the low Reynolds number environment, effect of inertia force $m \ddot{x}$ can be ignored. When the moving speed of sphere increases, drag force increases and became equal to trapping force. Once, the microsphere escapes from the trap, that is the moment, drag force equals to trapping force. At that time, (1) becomes,

$$
F_{\text {drag }}=-F_{\text {trap }}(x)
$$

Combining (4) with (2) and (3):

$$
-6 \pi \eta r \dot{x}=-k x
$$

Thus, maximum speed that target microsphere can achieve depends on the trapping force $(k x)$, viscosity of the medium $(\eta)$ and the size of the sphere $(r)$ as shown below:

$$
\dot{x}=\frac{k x}{6 \pi \eta r}
$$

Stokes' law explains the drag force exerted on micro spherical shapes. However, deriving the drag force for arbitrary shape in micro-scale is an arduous task. Therefore, to analyse achievable maximum speed of each design, performance test in aqua environment was conducted.

Each microhand was trapped by optical tweezers. During the trapping task, the orientation control of the half-cylinder shape microhand was more challenging compared to other two designs as it was non-spherical and asymmetric structure. Trapping was achieved for all three microhands.

After a successful trapping, transportation task was conducted. As an example, transportation of the cylinder with sphere microhand can be seen in Fig. $7(a, b, c)$ in the 


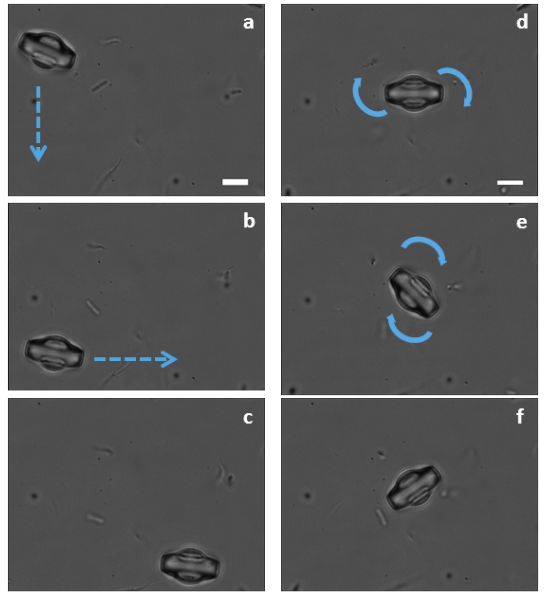

Fig. 7. Microhand transportation $(\mathrm{a}, \mathrm{b}, \mathrm{c})$ and rotation $(\mathrm{d}, \mathrm{e}, \mathrm{f})$ (white bar: 5 $\mu \mathrm{m})$.

visible space of $40 \times 50 \mu \mathrm{m}$. Transportation in $3 \mathrm{D}$ for each microhand was carried out successfully. Then, rotation of each microhand by using multi-spot laser beam on them was achieved as seen in Fig. 7 (d,e,f). By transportation and rotation tasks, manipulability of each microhand was verified under optical tweezers.

The maximum achievable speed for each microhand was investigated. By using AOD, the location of laser beam spot on horizontal plane was altered. The speed of spot relocation was increased step by step to find out at what speed microobjects would fail to follow the trap. The half cylinder, the sphere and the cylinder with sphere microhands were transported with a maximum speed of $120 \mu \mathrm{m} / \mathrm{s}, 190$ $\mu \mathrm{m} / \mathrm{s}$ and $180 \mu \mathrm{m} / \mathrm{s}$, respectively.

\section{B. MANIPULATION OF THE CHALLENGING OBJECTS}

To support the proposed idea of manipulation ability enhancement for optical tweezers, two different non-spherical, large size microobjects were chosen as target objects.

First, a rectangle shape $(15 \times 20 \times 4 \mu \mathrm{m})$ object manipulated by using a pair of same kind of microhand as an end effector of optical tweezers. Fig. 8 (a,b,c) shows manipulation of the rectangle target by two cylinder with sphere microhands. Microhands approached to target object from two opposite direction and grasped it. Then, target object was transported from center to out of visible space.

In the Fig. 8 (d,e,f), rotation of the same rectangle target was conducted successfully. The target object was grasped using two microhands. Then rotated $360^{\circ}$.

Same transportation and rotation tasks were carried out for cross shape target object $(15 \times 20 \times 4 \mu \mathrm{m})$, first without using any microhand (direct manipulation) and then using three different microhands for indirect manipulation to be able to compare direct and indirect manipulation of arbitrary shapes. Due to large size and flat surface features of cross shape target object, direct manipulation attempts failed each time. In every attempt, exposing laser light on target object, pushed the object away in-z axis. After that, indirect manipulation
TABLE I

PERFORMANCE OF EACH MICROHAND.

\begin{tabular}{ccccc}
\hline \hline & direct & $\begin{array}{c}\text { half } \\
\text { cylinder }\end{array}$ & sphere & $\begin{array}{c}\text { cylinder } \\
\text { with sphere }\end{array}$ \\
\hline Max.Speed $(\mu \mathrm{m} / \mathrm{s})$ & - & 120 & 190 & 180 \\
Success Rate & $0 \%$ & $50 \%$ & $90 \%$ & $85 \%$ \\
\hline
\end{tabular}

task was carried out for cross shape target as seen in Fig. 9. For these manipulation tasks, each microhand kind was used as a pair and success rate was sought. Out of 20 trial for each microhands, 10 times for the half cylinder, 18 times for the sphere an 17 times for the cylinder with sphere were found as a successful attempts. In this case, success is the transportation and rotation of target objects without loosing the traps of microhands in z-axis. See the attachment video for the experiment results.

\section{DISCUSSION}

Manipulation ability of optical tweezers depends on the target object features. To extend the manipulation capability of optical tweezers, designing various microhands as end effectors of optical tweezers is proposed. Hence, manipulation of microobjects may depend less on objects' features.

In this study, developed microhands were trapped, transported and rotated one-by-one to verify manipulability of these microstructures using optical tweezers. Then, manipulation of challenging microobjects, which represents nonspherical, asymmetric, large size, unsuitable refractivity and conductive objects, was achieved to support the idea of manipulation ability enhancement of optical tweezers.

During the speed test of microhands, the half cylinder shape was the slowest among three designs as seen in Table I. Two possible reasons for that are shape was not efficient in terms of drag force in liquid and refractivity of this nonspherical shape was not strong enough for trapping at higher speeds.

During manipulation of challenging microobjects, as seen in Table I, direct manipulation attempts failed every time. For indirect manipulation trials, using sphere and cylinder with sphere showed similar results as they both posses spherical shape where the half cylinder shape had lower success rate due to weak trapping strength.

Approaching from two opposite sides of a target object was not the only way to transport objects in 2D. As seen in Fig. 10, transportation of rectangle shape target object using two sphere microhands from the same side was another way of manipulation.

As manipulation success rates of the sphere and the cylinder with sphere shape microhands were similar, a new structure which is the combination of spherical and nonspherical parts will be studied to manipulate various objects in $3 \mathrm{D}$ as a future work. Indirect manipulation in $3 \mathrm{D}$, particularly, in z-axis with sphere shape is not convenient. Hence, using non-spherical shapes with spherical trapping 

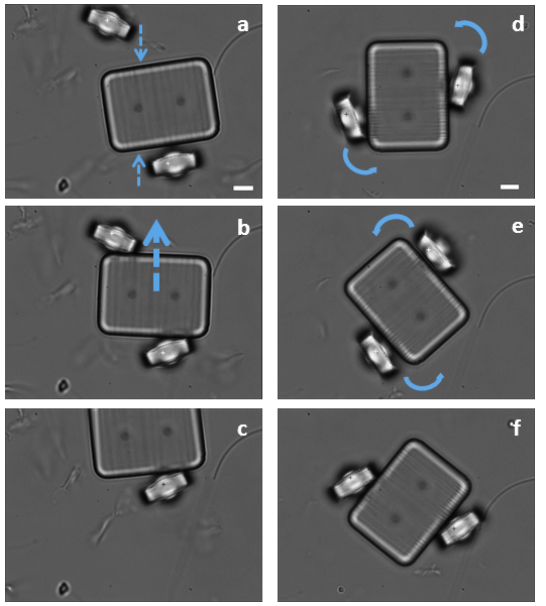

Fig. 8. Rectangle shape target object transportation (a,b,c) and rotation (d,e,f) using multi-microhands, (white bar: $5 \mu \mathrm{m}$ ).
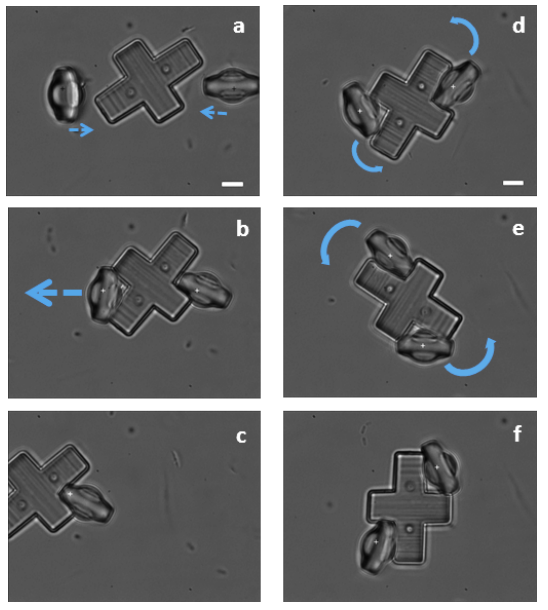

Fig. 9. Cross shape target object transportation (a,b,c) and rotation (d,e,f) using multi-microhands, (white bar: $5 \mu \mathrm{m}$ ).

point would be a solution for 3D indirect manipulation of microobjects. Furthermore, image processing for automation of indirect manipulation task will be investigated as a future work.

\section{REFERENCES}

[1] D. G. Grier, A revolution in optical manipulation, Nature, vol. 424, no. 6950, pp.810-816, 2003.

[2] F. Arai, K. Yoshikawa, T. Sakami, and T. Fukuda, Synchronized laser micromanipulation of multiple targets along each trajectory by single laser, Applied Physics Letters, vol. 85, no. 19, pp.4301-4303, 2004.

[3] A. Ashkin, Acceleration and trapping of particles by radiation pressure, Phys. Rev. Lett., vol. 24, no.4, pp.156-159 1970.

[4] A. Ashkin, J. M. Dziedzic, J. E. Bjorkholm, and S. Chu, Observation of a single-beam gradient force optical trap for dielectric particles, Opt. Lett., vol. 11, no. 5, pp.288-290, 1986.

[5] S. Hu, and D. Sun, Automatic transportation of biological cells with a robot-tweezer manipulation system, The International Journal of Robotics Research, vol. 30, no. 14, pp.1681-1694, 2011.

[6] M. W. Berns, Y. Tadir, H. Liang, and B. Tromberg, Laser scissors and tweezers, Methods Cell Biol. vol. 55, pp.71-98, 1998.

[7] C. Bustamante, S. B. Smith, J. Liphardt, and D. Smith, Singlemolecule studies of DNA mechanics, Current opinion in structural biology, vol. 10 no. 3, pp.279-285, 2000.
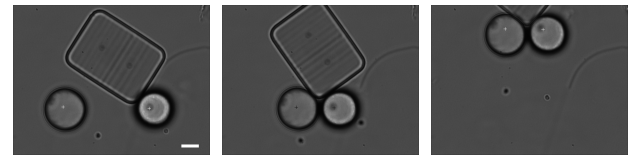

Fig. 10. An alternative manipulation method using two ball microhands from the same side of the target object,(white bar: $5 \mu \mathrm{m}$ ).

[8] T. N. Buican, M. J. Smith, H. A. Crissman, G. C. Salzman, C. C. Stewart, and J. C. Martin, Automated single-cell manipulation and sorting by light trapping, Applied Optics, vol. 26, pp.5311-5316, 1987.

[9] R. Steubing, S. Cheng, W. H. Wright, Y. Numajiri, and M. W. Berns, Laser induced cell fusion in combination with optical tweezers: the laser cell fusion trap, Cytometry, vol. 12, pp.505-510, 1991.

[10] X. Gou, H. Yang, T. M. Fahmy, Y. Wang, and D. Sun, Direct measurement of cell protrusion force utilizing a robot-aided cell manipulation system with optical tweezers for cell migration control, The International Journal of Robotics Research, vol. 33, no. 14, pp 1782-1792, 2014.

[11] Y. Tan, C. W. Kong, S. Chen, S. H. Cheng, R. A. Li, and D. Sun, Probing the mechanobiological properties of human embryonic stem cells in cardiac differentiation by optical tweezers, Journal of biomechanics, vol. 45 , no. 1, pp.123-128, 2012

[12] F. Arai, T. Endo, H. Maruyama, T. Fukuda, T. Shimizu, S. Kamiya, 3D Manipulation of lipid nanotubes using laser trapped functional gel microbeads, IEEE/RSJ International Conference on Intelligent Robots and Systems (IROS), San Diego, USA, pp.3125-3130, 2007.

[13] H. Maruyama, R. Iitsuka, K. Onda, and F. Arai, Massive parallel assembly of microbeads for fabrication of microtools having spherical structure and powerful laser manipulation, IEEE International Conference on Robotics and Automation (ICRA), pp.482-487, 2010.

[14] J. Glckstad, Optical manipulation: Sculpting the object, Nature Photonics, vol. 5, pp.7-8, 2011.

[15] F. Arai, H. Maruyama, T. Sakami, A. Ichikawa, and T. Fukuda, Pinpoint injection of microtools for minimally invasive micromanipulation of microbe by laser trap, IEEE/ASME Transactions on Mechatronics, vol. 8, no. 1, pp.3-9, 2003.

[16] H. Maruyama, T. Fukuda, and F. Arai, Functional gel-microbead manipulated by optical tweezers for local environment measurement in microchip, Microfluidics and Nanofluidics, vol. 6, no. 3, pp. 383-390, 2009

[17] D. B. Phillips, J. A. Grieve, S. N. Olof, S. J. Kocher, R. Bowman, M J. Padgett, M. J. Miles, and D. M. Carberry, Surface imaging using holographic optical tweezers, Nanotechnology, vol. 22, no. 28, pp. 285503, 2011.

[18] S. Fukuda, K. Onda, H. Maruyama, T. Masuda, and F. Arai, 3D Fabrication and Manipulation of Hybrid Nanorobots by Laser, IEEE International Conference on Robotics and Automation (ICRA), pp.2594 2599,2013

[19] A. Thakur, S. Chowdhury, P. vec, C. Wang, W. Losert, and S. K. Gupta, Indirect pushing based automated micromanipulation of biological cells using optical tweezers, The International Journal of Robotics Research (IJRR), vol. 33, pp.1098-1111, 2014.

[20] S. Kawata, H.-B. Sun, T. Tanaka, and K. Takada, Finer features for functional microdevices, Nature, vol. 412, pp.697-698, 2001.

[21] E. Avci, H. Yabugaki, T. Hattori, K. Kamiyama, M. Kojima, Y. Mae, and T. Arai, Dynamic releasing of biological cells at high speed using parallel mechanism to control adhesion forces, IEEE International Conference on Robotics and Automation (ICRA), pp.3789-3794, 2014.

[22] A. T. O'Neil, I. MacVicar, L. Allen, M. J. Padgett, Intrinsic and extrinsic nature of the orbital angular momentum of a light beam, Phys. Rev. Lett., vol. 88, no. 5, pp.053601, 2002.

[23] D. Ruh, B. Trankle, and A. Rohrbach, Fast parallel interferometric $3 \mathrm{~d}$ tracking of numerous optically trapped particles and their hydrodynamic interaction, Optics Express, vol. 19, no. 22, pp.2162721642, 2011

[24] M. C. Zhong, X. B. Wei, J. H. Zhou, Z. Q. Wang, and Y. M. Li, Trapping red blood cells in living animals using optical tweezers, Nat. Commun., 4:1786, 2013. 